\title{
A contextualisation approach to health promotion guideline development in South Africa
}

\author{
N Siegfried, ${ }^{1}$ MB ChB, MPH Hons, FCPHM (SA), DPhil; B Draper, ${ }^{2}$ MB ChB, MMed (PH), PGDip Psych; \\ G Draper, ${ }^{2} \mathrm{MB}$ ChB, MSc (Mother and Child Health), FCOG (SA), MPhil (Higher Education), PGDip Educational Technology; \\ M Porter, ${ }^{3}$ MB ChB, MPH; C Bonaconsa, ${ }^{4}$ BCur, MSc (Nursing); J Hunter, ${ }^{5}$ BA (Cur), PGDip Health Services Management, MBA; \\ L Moeng-Mahlangu, ${ }^{6} \mathrm{MSc}$ (Dietetics); S Asmall, ${ }^{7} \mathrm{MB} \mathrm{ChB}$
}

${ }^{1}$ Alcohol, Tobacco and Other Drug Research Unit, South African Medical Research Council, Cape Town, South Africa

2 Private consultant, Cape Town, South Africa

${ }^{3}$ Independent researcher and public health consultant, Elliotdale, South Africa

${ }^{4}$ Department of Paediatrics and Child Health, Faculty of Health Sciences, University of Cape Town, South Africa

${ }^{5}$ Primary Health Care, National Department of Health, Pretoria, South Africa

${ }^{6}$ Health Promotion and Nutrition, National Department of Health, Pretoria, South Africa

${ }^{7}$ Health System Strengthening: Primary Health Care, National Department of Health, Pretoria, South Africa

Corresponding author: N Siegfried (nandi.siegfried@mrc.ac.za)

Background. Risk factors for chronic illness contribute significantly to the disease burden in South Africa. The National Department of Health $(\mathrm{NDoH})$ commissioned the development of a toolkit of health promotion guidelines for use by healthcare professionals working in the primary care setting to address this burden.

Objectives. To (i) demonstrate the contextualisation approach to evidence-based health promotion recommendations; and (ii) present the development process of a contextually sensitive and illustrated fit-for-purpose product.

Methods. A contextualised approach was used whereby evidence from rigorous guidelines produced elsewhere was tailored to local conditions. The scope of the toolkit included five risk factors and 22 conditions identified by the NDoH and was underpinned by the Theory of Planned Behaviour. Potential health promotion messages relevant to risks, conditions or both were formulated as population, intervention, comparison and outcome (PICO) questions. The team searched for and selected evidence for each PICO question in a stepwise hierarchical manner and categorised sources as: (i) World Health Organization (WHO) guidelines; (ii) Cochrane systematic reviews; and (iii) non-Cochrane systematic reviews. Those messages supported by source-based evidence were included in the toolkit with culturally appropriate illustrations. Regular engagement with stakeholders included an initial health department stakeholder consultation, a focus group with national programme managers on the appearance and content of a draft toolkit, and a presentation of the final draft at a forum of provincial managers. Final approval of the toolkit rested with programme representatives.

Results. A total of 152 PICO questions were formulated. Supporting evidence was identified from 42 current WHO guidelines and 45 Cochrane systematic reviews to answer 147 PICO questions with several guidelines relevant to more than one risk or condition. Evidence for a further five PICO questions was obtained from non-Cochrane systematic reviews. Six additional service delivery messages and four 'no harm' messages were included to align the toolkit with current national guidelines. The illustrated toolkit was well received by stakeholders nationally and provincially, with programme managers expressing a high degree of willingness to adopt a preventive approach in the primary care clinic setting.

Conclusions. Use of a tailored contextualised approach to health promotion guidelines resulted in a culturally appropriate tool based on evidence gathered from rigorous sources and probably reduced development time and costs. Adherence to a robust framework to identify evidence ensured that the toolkit conforms to international guideline development standards.

S Afr Med J 2018;108(12):1036-1041. DOI:10.7196/SAMJ.2018.v108i12.13129

South Africa (SA) faces a quadruple burden of disease comprising communicable, non-communicable, perinatal and maternal, and injury-related disorders. ${ }^{[1]}$ In 2010, the highest proportion of disability-adjusted life-years lost in SA was attributable to preventive factors including alcohol use, high body mass index, high blood pressure, dietary risks and smoking (when unsafe sex is excluded as a separate risk factor). ${ }^{[2]}$

SA has adopted the principles of universal health coverage, and the introduction of national health insurance in the country is anticipated. ${ }^{[3]}$ Within this policy arena, there is a shift from a curative, facility-centred service delivery model of healthcare to preventive health promotion through patient empowerment. ${ }^{[4]}$ Several condition-based national guidelines incorporate health promotion messages, but no national guidelines for health promotion in primary care exist. In 2015, to address this gap, the National Department of Health $(\mathrm{NDoH})$ commissioned the development of a toolkit of health promotion guidelines specifically for use in primary care.

Guideline development methodology has gained increasing prominence in the knowledge translation field over the past decade. Guidelines are ideally created through a process of expert public consultation following evidence collation and interpretation in the context of other factors including priority, affordability, feasibility and acceptability. ${ }^{[5]}$ While such processes may lead to rigorous and fit-for-purpose guidelines, the process is often lengthy and 
expensive. Several alternative approaches to guideline formulation have been defined, including: ( $i$ ) adoption of guidelines already in existence with limited changes to the key messages prior to implementation; (ii) contextualisation, where guidelines produced elsewhere can be adopted but additional information is required to fit local conditions; and (iii) adaptation, where guidelines produced elsewhere are amended to include local research evidence and expert group consensus. ${ }^{[6-8]}$ Alternative approaches are likely to provide more efficient use of resources and have particular applicability in resource-constrained settings. A further approach which combines adoption, adaptation and de novo guidelines development has recently been described and labelled adolopement. ${ }^{[9]}$

\section{Objectives}

In this article we demonstrate how we tailored the methodology of a guidelines contextualisation approach to ensure that the health promotion recommendations contained in the primary care Health for All toolkit were evidence based, of high quality and up to date, and relevant to SA. We further present the parallel process undertaken to develop a contextually sensitive and illustrated fit-forpurpose product for primary care providers and their clients.

\section{Methods}

\section{Scope}

\section{Inclusion criteria}

Before development commenced, the $\mathrm{NDoH}$ specified that the toolkit conform to the following: (i) be a clinical tool for use by health professionals at primary care facilities; (ii) be used in oneon-one consultation with presenting clients; (iii) focus on five key risks of chronic illness, namely diet and nutrition, lack of physical activity, tobacco use, harmful alcohol and drug use, and unsafe sexual behaviours; and (iv) align with specific current programme policies and existing national clinical guidelines.

At the time of development of the toolkit, a guideline for the management of clinical conditions, Primary Care 101 (PC101), was already well established in SA primary care facilities. ${ }^{[10]}$ The intention of the toolkit was to supplement the clinical guidelines by identifying and mitigating risk in both healthy clients and those with existing conditions. The toolkit would equip healthcare workers with tools to guide clients to live well and prevent further complications within their own locus of control.

During the development process, the $\mathrm{NDoH}$ expanded the scope beyond a risk-based approach to include a conditions-based health promotion approach to 22 common communicable and chronic noncommunicable diseases, mental health, sexual reproductive health, cancer and oral health (Table 1).

\section{Exclusion criteria}

Activities that form part of regular provision of healthcare services, either by the healthcare professional directly or by the broader health system, were excluded from the development process. For example, a recommendation that a patient with tuberculosis should seek HIV counselling and testing was considered an action that the treating healthcare professional should encourage and arrange for referral, rather than an action that the patient would be encouraged to pursue on their own. Upstream determinants of health were also not considered, e.g. regulations to restrict salt in foodstuffs or environmental risks.

\begin{tabular}{|c|c|}
\hline Risks & Conditions and specific health areas \\
\hline Unhealthy eating, overweight and obesity & Communicable \\
\hline Alcohol and harmful drug use & HIV and AIDS \\
\hline Unsafe sex & Tuberculosis \\
\hline Lack of physical activity & Sexually transmitted infections \\
\hline \multirow{23}{*}{ Tobacco use and smoking } & Non-communicable \\
\hline & Hypertension \\
\hline & Diabetes \\
\hline & Cardiovascular disease \\
\hline & Asthma \\
\hline & Chronic obstructive pulmonary disease \\
\hline & Epilepsy \\
\hline & Chronic bone and joint disease \\
\hline & Mental health \\
\hline & Depression \\
\hline & Anxiety \\
\hline & Stress and trauma \\
\hline & Psychosis \\
\hline & Dementia \\
\hline & Sexual health \\
\hline & Women's sexual health \\
\hline & Men's sexual health \\
\hline & Maternal: antenatal health \\
\hline & Maternal: postnatal health \\
\hline & Menopause \\
\hline & Other \\
\hline & Cancer \\
\hline & Oral health \\
\hline
\end{tabular}




\section{Establishing underlying principles}

An initial literature review was conducted to identify appropriate and effective health promotion strategies and principles to underpin the toolkit. These were shared and agreed with the NDoH prior to commencement of the project and included: $(i)$ recognition of the importance of self-management; (ii) visual representation of risk; (iii) cultural appropriateness; and (iv) the promotion of health literacy. As there are several theories of behavioural change, we identified the Theory of Planned Behaviour to inform and guide development of the toolkit. ${ }^{[11]}$ According to the model, behavioural intention is primarily influenced by a person's attitude and subjective norms, but recognises that perceived behavioural control also modifies intention. Based on systematic review evidence, motivational interviewing techniques incorporating those used in brief interventions were selected as the communication method to deliver the toolkit recommendations. ${ }^{[12]}$

The principles of evidence-based healthcare to promote rigorous research methods to identify and appraise all available evidence formed the theoretical foundation for the inclusion of recommendations. ${ }^{[13]}$ Specifically, we recognised that guidelines based on well-conducted systematic reviews should form the basis of all included recommendations.

\section{Appearance of the toolkit}

The NDoH requested that the appearance of the toolkit reflect other guidelines already in use in primary care. Additionally, the toolkit would stand on the desk with a page of detailed informative text facing the provider and a page of illustrated messages facing the patient for each risk and condition. Premised on individuals' limited capacity to recall information and comply with only a limited number of resolutions, ${ }^{[14]}$ five actionable ('to do') messages would be linked to each risk and condition. Healthcare providers would be encouraged to present a maximum of two 'to do' messages to a patient for their consideration. Illustrations would be used to depict all the included health promotion messages and would be culturally sensitive to improve comprehension and recall of messages. ${ }^{[15]}$ The development team contracted a graphic designer familiar with the healthcare setting to conceptualise and produce the appearance and illustrations within a typical SA local community thematic.

\section{Evidence application}

\section{Question formulation}

At the outset, the development team generated a list of health promotion messages sourced from current national primary care guidelines, the Essential Drugs List and condition-specific guidelines developed by local clinical associations (list available from authors). In addition, health promotion messages in the SA managed healthcare sector were sourced and a search for countrylevel health promotion guidelines was conducted. Those considered relevant to the SA context were included, and were primarily Canadian and Australian health promotion guidelines with a focus on indigenous populations. ${ }^{[16-18]}$

The team supplemented the above with messages drawn from their extensive clinical experience (including primary healthcare, certain medical specialties, nursing, dietetics and public health), and engagement with peer networks to develop a matrix of health promotion messages linked to each of the identified risks and conditions (Fig. 1). Together the members of the development team then systematically evaluated each of the messages for the following: (i) did the message include an 'actionable' intervention; and (ii) was it theoretically possible for the client to adopt the intervention activity into their lives.

If the above criteria were met, the message was reformulated as a research question using the PICO format $(\mathrm{P}=$ population, $\mathrm{I}=$ intervention, $\mathrm{C}=$ comparison, $\mathrm{O}=$ outcome). For many messages, it was necessary first to articulate whether the intervention itself was effective and then secondly to identify whether promoting the intervention was effective. For example, a message to promote exercise in people with osteoarthritis required identification of both whether physical activity is effective for osteoarthritis and if so, whether promotion of the physical activity is effective. Within the PICO question, the specific primary outcome also required articulation. The final PICO question was therefore reformulated as: 'Does physical activity (I) and promotion thereof compared with no activity $(\mathrm{C})$ reduce the pain $(\mathrm{O})$ experienced by people with osteoarthritis $(\mathrm{P})$ ?'

\section{Evidence algorithm}

For each PICO, we searched for evidence using a predetermined algorithm in a stepwise hierarchical approach to determine inclusion or exclusion of each health message.

Step 1 involved a search of World Health Organization (WHO) content webpages mapped to each of the five risks or 22 conditions to identify guidelines (2010 onwards) that used the Grading of Recommendations Assessment, Development and Evaluation (GRADE) approach to formulate recommendations. ${ }^{[5]}$ WHO guidelines are aimed at low- and middle-income countries and are therefore likely to be relevant to the SA setting. The GRADE approach is a widely used method to appraise overall quality of evidence that includes evaluation of risk of bias, imprecision, indirectness and inconsistency in evidence syntheses. ${ }^{[19]}$ It is a useful indicator of the rigour of guideline development. Where a recommendation relevant to the $\mathrm{PICO}$ was identified in a $\mathrm{WHO}$ guideline, it was marked for possible inclusion in the toolkit and no further search was undertaken; if no recommendation was identified, step 2 was activated.

Step 2 involved a search of the Cochrane Library for relevant Cochrane systematic reviews (2010 onwards) using search terms for the risks and conditions. Cochrane reviews are widely recognised as the gold standard of systematic reviews of effectiveness, ${ }^{[20]}$ and conducting a GRADE assessment ${ }^{[19]}$ of the quality of the evidence is mandatory. If evidence was available from a Cochrane review to inform the PICO, it was marked for possible inclusion; if no relevant review was identified, step 3 was undertaken.

Step 3 comprised a comprehensive search of the PubMed and Embase databases using a syntax strategy of terms related to the intervention and/or population in the PICO and filtered by study design (systematic review and randomised controlled trial). Where relevant non-Cochrane systematic reviews of effectiveness were identified, we selected the review based on the most recent date of publication combined with the presence of randomised controlled trials in the review study selection criteria and use of a quality appraisal approach to rate the evidence, such as GRADE or a comparable approach. If more than one review met the criteria or if reviews included different studies, we applied the AMSTAR tool to each review to appraise its quality, and selected the review of higher quality for inclusion. ${ }^{[21]} \mathrm{Had}$ no reviews been identified, we had planned to conduct a de novo systematic review, but in the event this was not necessary.

We contacted technical leads at the WHO directly when uncertainties existed as to the inclusion of GRADE in a WHO guidelines process. We also contacted authors of Cochrane reviews 
and Cochrane editors to access review results if the review was identified at the protocol stage.

Following on from the stepwise selection process, a separate evidence source document was compiled in tabular form, colourcoded by source type and including the recommendation or message as presented in the evidence source, the reference(s), the level of evidence, and alignment with national guidelines where relevant.

\section{Selection of health promotion message}

A maximum of five key health promotion ('to do') messages were selected for each risk and condition. Messages were prioritised according to quality of the evidence (high-quality effectiveness data were preferable), relevance to the local setting, and feasibility including affordability to the client. Where more than five messages existed per risk or condition, additional messages were included only when considered vital in addressing the burden of disease. Final selection was achieved through consensus within the development team.

\section{Engagement with stakeholders}

The development-feedback timeline included: ( $i$ ) an internal national departmental key stakeholder engagement meeting and focus group held in May 2015 to finalise the scope and proposed toolkit format; (ii) a consultation with national departmental programme managers in August 2015, where a draft version of the toolkit, including a sample graphic of a condition and risk section, was presented and written feedback was invited; and (iii) a national forum comprising national and provincial departmental representatives in November 2015, where the final draft of the toolkit was showcased and commentaries were invited.

\section{Ethical considerations}

This article reports a development process commissioned by the $\mathrm{NDoH}$, and ethics approval was not required.

\section{Results}

\section{Evidence application}

A total of 152 PICO questions were formulated. We identified evidence from 42 current WHO guidelines and 45 Cochrane systematic reviews to answer 147 PICO questions (several guidelines were relevant to more than one risk or condition). As no evidence was forthcoming from WHO guidelines or Cochrane reviews for five PICO questions, we conducted comprehensive searches of PubMed and Embase to identify evidence from non-Cochrane systematic reviews of effectiveness in support of each PICO question (Table 2). ${ }^{[22-26]}$

In addition to the PICO-based health messages, there were six messages that were classified as service delivery messages (for example, 'Know your HIV status') and four that were included as 'no harm' messages upon request by the national department to ensure alignment with national guidelines.

Several health promotion messages were linked to more than one risk or condition. For example, a message to increase physical activity was pertinent to the risk factor of lack of physical activity, overweight and obesity, and for multiple conditions including hypertension, cardiovascular disease and diabetes. Therefore, in the final format, the toolkit includes 34 discrete 'to do' health promotion messages for the risk factors and 98 discrete 'to do' messages for the included conditions.

\section{Engagement with stakeholders}

At the first focus group held within the $\mathrm{NDoH}$, stakeholders requested that consideration be given to the socioeconomic and cultural context of users of the health services during the development of the toolkit. They also requested that the toolkit be illustrated, colourful and user friendly. These concerns were addressed prior to the presentation of the draft toolkit at the consultation with programme managers within the $\mathrm{NDoH}$, many of whom provided written feedback on sections relevant to their programmes.

At the national forum where the final draft was showcased, over $90 \%$ of 95 participants felt that the toolkit was contextually appropriate (Fig. 1) and supported the shift in approach from a

Table 2. Results for the searches for systematic reviews for five PICO questions for which no World Health Organization guidelines or Cochrane evidence were identified

\begin{tabular}{|c|c|c|c|c|c|}
\hline PICO question & $\begin{array}{l}\text { Number of } \\
\text { PubMed } \\
\text { records }\end{array}$ & $\begin{array}{l}\text { Number } \\
\text { of Embase } \\
\text { records }\end{array}$ & $\begin{array}{l}\text { Number } \\
\text { of possibly } \\
\text { eligible } \\
\text { reviews } \\
\text { identified }\end{array}$ & Final review reference & $\begin{array}{l}\text { AMSTAR } \\
\text { rating }\end{array}$ \\
\hline $\begin{array}{l}\text { Does palliative support including communication and } \\
\text { engagement improve the quality of life of adult men and } \\
\text { women with terminal illness? }\end{array}$ & 263 & 305 & 6 & Ventura et al. ${ }^{[22]}$ & $9 / 11$ \\
\hline $\begin{array}{l}\text { Does promotion to reduce sweetened drinks intake compared } \\
\text { with no promotion reduce body weight in adult men and } \\
\text { women? }\end{array}$ & 172 & 195 & 5 & Zheng et al. ${ }^{[23]}$ & $7 / 11$ \\
\hline $\begin{array}{l}\text { Does promotion of physical activity compared with no } \\
\text { promotion reduce problematic alcohol and substance use? }\end{array}$ & 165 & 69 & 6 & Wang et al. ${ }^{[24]}$ & $8 / 11$ \\
\hline $\begin{array}{l}\text { Does promotion of weight loss compared with no promotion } \\
\text { reduce the pain of osteoarthritis in adult men and women? }\end{array}$ & 633 & 252 & 4 & Maly and Robbins ${ }^{[25]}$ & $9 / 11$ \\
\hline $\begin{array}{l}\text { Does promotion of alcohol cessation or reduction compared } \\
\text { with no promotion reduce impotence and erectile dysfunction } \\
\text { in adult men? }\end{array}$ & 81 & 85 & $\begin{array}{l}18 \text { (11 in } \\
\text { adult men; } \\
7 \text { specifi- } \\
\text { cally for } \\
\text { men living } \\
\text { with HIV) }\end{array}$ & Scott-Sheldon et al..$^{[26]}$ & $8 / 11$ \\
\hline
\end{tabular}




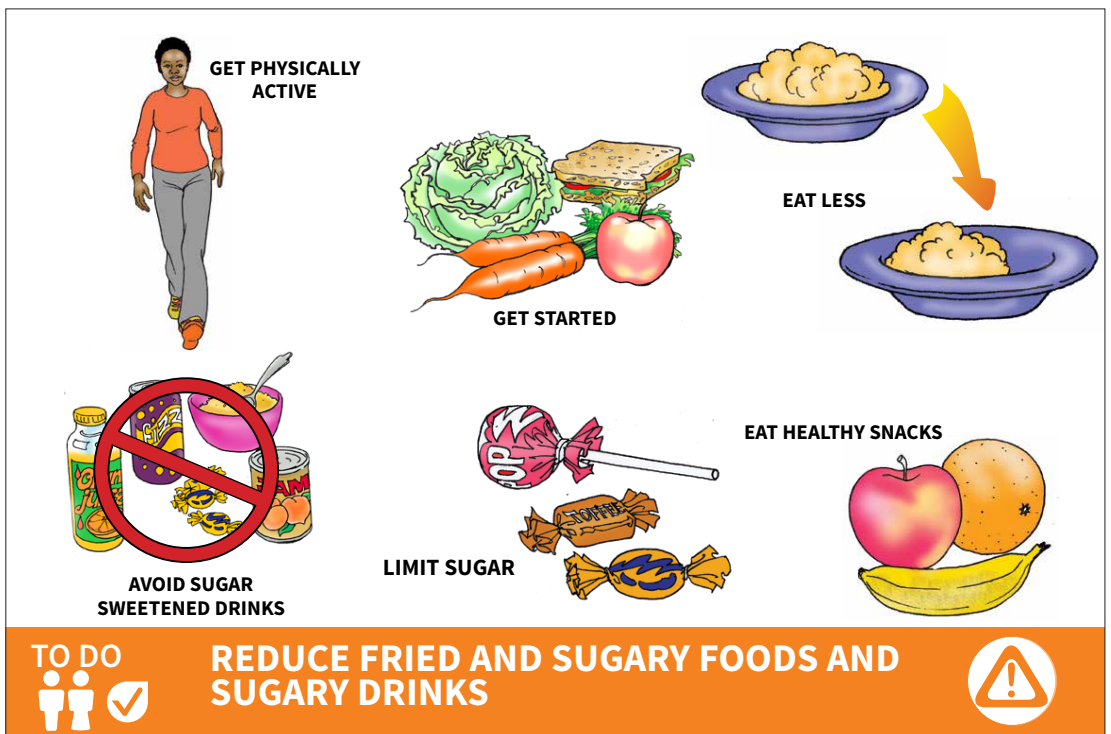

Fig. 1. Example of an illustrated page of health promotion 'to do' messages from the Health for All toolkit.

disease model to a risk-based approach to achieving a healthy lifestyle. Participants raised two challenges to the implementation of the toolkit: (i) language was limited to English, which is not the first language of most presenting clients; and (ii) overburdening staff with the introduction of a new guideline into a resource-strapped system. Despite these concerns, there was widespread support from directors and programme managers from all nine provinces for embedding health promotion and health risk mitigation within the overall responsibility of healthcare providers.

\section{Discussion \\ Main findings}

The Health for All health promotion toolkit is the first national comprehensive suite of health promotion guidelines aimed at addressing both health risk behaviours and health conditions of clients presenting to primary care facilities. As such it allows healthcare providers to identify and mitigate risk, even in healthy patients, and serves as a guide to clients to live well with existing conditions and prevent further complications. Development of the toolkit employed a guidelines contextualisation approach to ensure that all health promotion messages were based on evidence retrieved through rigorous methods. The approach was tailored so that messages selected for inclusion were based on feasibility and relevance to the local context in SA.

\section{Strengths and limitations}

Use of a predefined hierarchical process to select evidence minimised selection bias within the development team. Importantly, by reformulation of the messages as PICO questions, the development team adopted a state of 'equipoise' with respect to evidence retrieval, rather than seeking to source evidence in support of any particular health message. At times this was a challenge, given that certain messages already present in some national guidelines appeared not to have a strong evidence base. Departmental pressure dictated that these were included in the toolkit to ensure harmonisation across guidelines. An agreement was brokered between the relevant programme managers and the development team to include those messages only if they were considered to do 'no harm' or were globally accepted as best practice and to mark them as such in the supportive materials. This illustrates the dynamic environment of guideline development and the need to acknowledge and manage the many competing interests and tensions present during the collaborative development process.

Through using recommendations made by the WHO and evidence from published Cochrane reviews, we reduced the need to conduct new systematic reviews, which can be costly and time intensive. The overall product has a far greater coverage of content than if we had developed messages for selected risks and conditions de novo, with the entire development process spanning less than a year. The success of the contextualisation approach is dependent on matching the evidence to local needs and presenting it within an appropriate context. It was therefore essential for the selected development team to have not only research skills but also sufficient prior experience of working in the clinic setting. The team worked closely with programme managers at the NDoH to ensure that the challenges of the primary health clinic environment were considered when formulating the messages and that the broader community was sensitively represented in the illustrations throughout. Formal focus group testing of the toolkit with clinic staff and patients in the community would further strengthen the development process, but was not undertaken owing to cost and organisational constraints.

Generation of the initial list of health promotion messages used an iterative approach, but some important messages may have been missed and therefore not included as PICO questions. This limitation was addressed by including engagement with multiple stakeholders, including peer networks, throughout the process. No formal guideline development panel was convened and tasked with scrutiny of the final decisions of the development team, and no formal feedback was requested from clinical associations, so there may be some omissions.

\section{Comparison with other health promotion guidelines}

In general, health promotion messages are included as a minor component of condition-specific clinical guidelines and play a supporting role to pharmacological recommendations. As far as we are aware, the toolkit is unique in its comprehensive coverage of risks and conditions common to primary care. In 2012 Denmark introduced national health promotion guidelines, which are risk based and aimed at implementation of activities within local communities rather than offering health promotion in the healthcare facility. ${ }^{[27]}$ In their 2015 article analysing the implementation challenges of the Danish guidelines, Rod and Hoybye ${ }^{[27]}$ claim that to their knowledge no other country has similar guidelines. We identified only one similar process to the Health for All toolkit, led by the Royal Australian College of General Practitioners in Australia. ${ }^{[28]}$ The Handbook of Non Drug Interventions (HANDI) project aims to promote effective non-drug treatments, making them visible and easy to use through an online formulary of non-drug interventions in healthcare, which have solid evidence of their effectiveness. The process to determine inclusion is made by an internal committee following topic selection, evidence assessment, judgement 
of relevance, general discussion and voting. For inclusion in HANDI, interventions must be supported by at least two positive good-quality randomised controlled trials with patient-relevant outcomes, or one trial with strong supportive evidence for the causal connection under investigation.

\section{Applicability of the toolkit}

The global burden of disease studies indicate a generalised trend towards non-communicable diseases replacing infectious diseases as drivers of morbidity across resource-constrained regions. ${ }^{[29]}$ As countries begin to recognise the contribution of environment, including social inequality and injustice, to disease clustering and health vulnerabilities, ${ }^{[30]}$ we predict that those risk factors that can be addressed by individual clients will become an increasingly important part of clinical practice and public health policy. We believe that the recommendations included in the toolkit are likely to have wide applicability in many low- and middle-income countries. Given the generic nature of most of the recommendations and the consideration given to accessibility and affordability of each health promotion message during toolkit development, it may be possible for countries to adopt the guidelines with minimal additional development. However, the presentation and format of the toolkit would need to be tailored to local context with appropriate illustrations and thematics.

\section{Next steps}

Implementation of the Health for All toolkit was initiated in June 2018 with training of provincial master trainers to lead training, uptake, and use in the provinces by primary healthcare providers. Training tools have been developed for this purpose and designated staff have received preliminary training. As the toolkit is scaled up across all provinces, there is opportunity for monitoring and evaluation of its uptake and usability by both staff and clients. Similar to other guidelines, the toolkit is a dynamic document and future revisions will be informed by feedback from users and local clinical associations. While the long-term effectiveness of the toolkit will be challenging to measure reliably, the toolkit provides a firm foundation for expansion of health promotion strategies beyond primary care.

\section{Conclusions}

Use of a tailored contextualised approach to health promotion guidelines development is likely to have reduced costs and time and resulted in a tool based on evidence gathered from rigorous sources. Choice of design and illustrations is key to ensuring that the guidelines are presented in a culturally appropriate format accessible to healthcare providers working in primary care. Future guidelines development teams are advised to select principles and a robust framework a priori to guide the development process and to ensure regular communication between all stakeholders. We believe the Health for All toolkit has the potential to begin to change the conversations within the primary care clinic.

\section{Declaration. None.}

Acknowledgements. The authors acknowledge the helpful comments of Ozayr Mohamed and Mohammed Jeenah on an earlier version of the manuscript.

Author contributions. SA conceptualised and led the Health for All project. $\mathrm{BD}, \mathrm{GD}, \mathrm{MP}, \mathrm{CB}, \mathrm{NS}$ and $\mathrm{SA}$ developed the toolkit, and evaluation thereof was conducted in partnership with $\mathrm{SA}, \mathrm{JH}$ and LM-M. NS prepared a draft manuscript and all authors contributed to revisions and approved the final version.
Funding. The development team (NS, BD, GD, MP, CB) was funded through Broadreach Healthcare with funding provided by the South African NDoH and the US President's Emergency Plan for AIDS Relief (PEPFAR). The Alcohol, Tobacco and Other Drug Research Unit of the South African Medical Research Council provided technical and financial support towards publication.

Conflicts of interest. None.

1. Mayosi BM, Lawn JE, van Niekerk A, Bradshaw D, Abdool Karim SS, Coovadia HM. Health in South Africa: Changes and challenges since 2009. Lancet 2012;380(9858):2029-2043. https://doi.org/10.1016/ S0140-6736(12)61814-5

2. Mayosi BM, Benatar SR. Health and health care in South Africa -20 years after Mandela. N Engl J Med 2014;371(14):1344-1353. https://doi.org/10.1056/NEJMsr1405012

3. Hofman KJ, McGee S, Chalkidou K, Tantivess S, Culyer AJ. National Health Insurance in South 3. Hofman Ke, McGee S, Chalkidou K, Tantivess S, Culyer AJ. National Health Insurance in South
Africa: Relevance of a national priority-setting agency. S Afr Med J 2015;105(9):739-740. https://doi. Africa: Relevance of a natio
org/10.7196/SAMJnew.8584

4. South African National Department of Health. White Paper on National Health Insurance, 4. South African National Department of Health. Whit
Government Gazette 11 December 2015;39506:1230.

Government Gazette 11 December 2015;39506:1230.
5. World Health Organization. WHO Handbook for Guideline Development. Geneva: WHO, 2014.

6. Dizon JM, Grimmer K, Louw Q, Kredo T, Young T, Machingaidze S. South African Guidelines Excellence (SAGE): Adopt, adapt, or contextualise? S Afr Med J 2016;106(12):1177-1178. https://doi. org/10.7196/SAMJ.2017.v106i12.11374

7. Graham ID, Harrison MB, Brouwers M, Davies BL, Dunn S. Facilitating the use of evidence in practice: Evaluating and adapting clinical practice guidelines for local use by health care organizations. J Obstet Gynecol Neonatal Nurs 2002;31(5):599-611. https://doi.org/10.1111/j.1552-6909.2002.tb00086.x

Wingood GM, DiClemente RJ. The ADAPT-ITT model: A novel method of adapting evidence-based HIV interventions. J Acquir Immune Defic Syndr 2008;47(Suppl 1):S40-S46. https://doi.org/10.1097/ QAI.0b013e3181605df1

9. Schunemann HJ, Wiercioch W, Brozek J, et al. GRADE Evidence to Decision (EtD) frameworks for adoption, adaptation, and de novo development of trustworthy recommendations: GRADEADOLOPMENT. J Clin Epidemiol 2017;81:101-110. https://doi.org/10.1016/.j.clinepi.2016.09.009

10. Fairall LR, Folb N, Timmerman V, et al. Educational outreach with an integrated clinical tool for nurse. Fairall LR, Folb N, Timmerman V, et al. Educational outreach with an integrated clinical tool for nurse-
led non-communicable chronic disease management in primary care in South Africa: A pragmatic cluster randomised controlled trial. PLoS Med 2016;13(11):e1002178. https://doi.org/10.1371/journal. pmed. 1002178

11. US Department of Health and Human Services. Theory at a Glance: A Guide for Health Promotion Practice. National Institute of Health, 2005. https://www.sbccimplementationkits.org/demandrmnch/ wp-content/uploads/2014/02/Theory-at-a-Glance-A-Guide-For-Health-Promotion-Practice.pdf (accessed 2 November 2018).

12. Lundahl B, Moleni T, Burke BL, et al. Motivational interviewing in medical care settings: A systematic review and meta-analysis of randomized controlled trials. Patient Educ Couns 2013;93(2):157-168. https://doi.org/10.1016/j.pec.2013.07.012

13. Brownson RC, Chriqui JF, Stamatakis KA. Understanding evidence-based public health policy. Am J Public Health 2009;99(9):1576-1583. https://doi.org/10.2105/AJPH.2008.156224

14. Cowan N. The magical number 4 in short-term memory: A reconsideration of mental storage capacity. Cowan N. The magical number 4 in short-term memory: A reconsideration of mer
Behav Brain Sci 2001;24(1):87-114. https://doi.org/10.1017/s0140525x01003922

15. Houts PS, Doak CC, Doak LG, Loscalzo MJ. The role of pictures in improving health communication: A Houts PS, Doak CC, Doak LG, Loscalzo MJ. The role of pictures in improving health communication:
A review of research on attention, comprehension, recall, and adherence. Patient Educ Couns A review of research on attention, comprehension, recall,

2006;61 (2):173-190. https://doi.org/10.1016/j.pec.2005.05.004
6. Health Canada. Aboriginal Diabetes Initiative Program Framework 2010 - 2015. Health Canada, 2011. http://www.healthcanada.gc.ca/ADI (accessed 5 June 2017).

17. Australian National Preventive Health Agency. Shape Up Australia Resources. Australian National Preventive Health Agency, 2014. http://www.quitnow.gov.au/internet/anpha/publishing.nsf/Content/ Shape+Up+Resources (accessed 5 June 2017).

18. Brady M, McKenzie-Taylor M. Talking About Alcohol with Aboriginal and Torres Strait Islander Patients: A Brief Intervention Tool for Health Professionals. Canberra: Commonwealth Department of Health and Ageing, 2003.

19. Guyatt GH, Oxman AD, Vist GE, et al. GRADE: An emerging consensus on rating quality of evidence and strength of recommendations. BMJ 2008;336(7650):924-926. https://doi.org/10.1136/ bmj.39489.470347.ad

20. Smith R. The Cochrane Collaboration at 20. BMJ 2013;347:f7383. https://doi.org/10.1136/bmj.f7383

21. Shea BJ, Grimshaw JM, Wells GA, et al. Development of AMSTAR: A measurement tool to assess the methodological quality of systematic reviews. BMC Med Res Methodol 2007;7:10. https://doi. the methodological quality of
org $/ 10.1186 / 1471-2288-7-10$

22. Ventura AD, Burney S, Brooker J, Fletcher J, Ricciardelli L. Home-based palliative care: A systematic literature review of the self-reported unmet needs of patients and carers. Palliat Med 2014;28(5):391402. https://doi.org/10.1177/0269216313511141

23. Zheng M, Allman-Farinelli M, Heitmann BL, Rangan A. Substitution of sugar-sweetened beverages with other beverage alternatives: A review of long-term health outcomes. J Acad Nutr Diet 2015;115(5):767-779. https://doi.org/10.1016/j.jand.2015.01.006

24. Wang D, Wang Y, Wang Y, Li R, Zhou C. Impact of physical exercise on substance use disorders: A meta-analysis. PloS One 2014;9(10):e1 10728. https://doi.org/10.1371/journal.pone.0110728

25. Maly MR, Robbins SM. Osteoarthritis year in review 2014: Rehabilitation and outcomes. Osteoarthr Cartil 2014;22(12):1958-1988. https://doi.org/10.1016/j.joca.2014.08.011

26. Scott-Sheldon LA, Carey KB, Cunningham K, Johnson BT, Carey MP. Alcohol use predicts sexual decision-making: A systematic review and meta-analysis of the experimental literature. AIDS Behav 2016;20(Suppl 1):S19-S39. https://doi.org/10.1007/s10461-015-1108-9

27. Rod MH, Hoybye MT. A case of standardization? Implementing health promotion guidelines in Rod MH, Hoybye MT. A case of standardization? Implementing health promotion gu
Denmark. Health Promot Int 2016;31(3):692-703. https://doi.org/10.1093/heapro/dav026

28. Royal Australian College of General Practitioners. The HANDI Project Australia 2017. http://www. racgp.org.au/your-practice/guidelines/handi/about/the-handi-project/ (accessed 18 May 2017).

29. GBD 2015 DALYs and HALE Collaborators. Global, regional, and national disability-adjusted life-years (DALYs) for 315 diseases and injuries and healthy life expectancy (HALE), 1990 - 2015: A systematic analysis for the Global Burden of Disease Study 2015. Lancet 2016;388(10053):16031658. https://doi.org/10.1016/S0140-6736(16)31460-X

30. Singer M, Bulled N, Ostrach B, Mendenhall E. Syndemics and the biosocial conception of health. Lancet 2017;389(10072):941-950. https://doi.org/10.1016/s0140-6736(17)30003-x 